\title{
Pedagogy-Driven Smart Games for Primary School Children
}

Fernando De la Prieta, Tania Di Mascio, Ivana Marenzi, and Pierpaolo Vittorini

\begin{abstract}
TERENCE is an FP7 ICT European project, highly multi-disciplinary, that is developing an adaptive learning system for supporting poor comprehenders and their educators. Their learning materials are stories and games, explicitly designed for classes of primary schools poor comprehenders, where classes were created via an extensive analysis of the context of use and user requirements. The games are specialised into smart games, which stimulate inference-making for story comprehension, and relaxing games, which stimulate visual perception and which train the interaction with devices (e.g., PC and tablet PC). In this paper we focus on how we used the pedagogical underpinnings and the acquired requirements to design the games of the system.
\end{abstract}

Keywords: Formalizations of pedagogical theories, serious games, game frameworks.

\section{Introduction}

More and more young children turn out to be poor (text) comprehenders: they demonstrate text comprehension difficulties related to inference-making skills, despite proficiency in word decoding and other low-level cognitive skills. Deep text

Fernando De la Prieta

University of Salamanca, Department of Computer Science, Spain

e-mail: fer@usal.es

Tania Di Mascio

University of L'Aquila, DISIM, Italy

e-mail: tania.dimascio@univaq. it

Ivana Marenzi

University of Hanover, L3S, Germany

e-mail: marenzi@13s.de

Pierpaolo Vittorini

University of L'Aquila, MeSVA, Italy

e-mail: pierpaolo.vittoriniacc.univaq.it

P. Vittorini et al. (Eds.): 2nd International Workshop on Evidence-Based TEL, AISC 218, pp. 33-41. DOI: 10.1007/978-3-319-00554-6_5 @ Springer International Publishing Switzerland 2013 
comprehension skills develop from the age of 7-8 until the age of 11, when children develop as in- dependent readers. Nowadays, there are several penciland-paper reading strategies for improving text reading comprehension, and specifically addressed to poor comprehenders, which could be delivered by an adaptive learning system (ALS), that is, a suite of functionalities designed to deliver, track, report on and manage learning content for specific learners [8][9].

TERENCE is a EU project -http://www.terenceproject.eu-- that aims at delivering the first ALS for enhancing the reading comprehension of poor comprehenders, building upon effective pencil-and- paper reading strategies, and framing them into a playful and stimulating environment. Learners are primary school poor comprehenders, hearing and deaf, older than 7 .

The goal of this paper is to explain how the playing material and tasks of TERENCE are designed and developed on top of an extensive analysis of the requirements of the TERENCE learners. First, the paper sets the groundwork by presenting the pedagogical theory and approach followed in TERENCE in Sec. 2. Then it outlines the types of data gathered for characterising the TERENCE learners and the analysed effective reading strategies and interventions for the TERENCE learners; in Sec. 3 and 4 is explained how the design and development of the TERENCE games, in particular, stems from such knowledge. For space limitations, we focus on the playing material, that is, games and playing tasks.

For information concerning the reading material and tasks, see [6]. Moreover, the models for the learning material and learners of the system are described in [4], how the user centered design (UCD) was used for them is in [2], whereas some of the adaptation rules are outlined in [5]. The game design for all the TERENCE games is in [3] and, finally, the architecture for games and their automatic generation is outlined in [10].

\section{The Pedagogical Underpinnings}

The theoretical framework underpinning of TERENCE is grounded on the constructivist pedagogical approach [15], which is a theory of learning that focuses on students being engaged in "doing", rather than passively engaged in "receiving" knowledge. In other words, constructivism states that learning takes place in contexts. This approach is committed to the general view that (1) learning is an active process of constructing rather than acquiring knowledge, and (2) instruction is a process of supporting that construction rather than communicating knowledge [11]. Nevertheless, knowledge does not simply arise from experience, but is build through experience over the current knowledge structures. The educator is required to orchestrate all the resources needed and must guide students in training them how to teach themselves [17].

Scaffolding is offered to the learner as an adequate environment where to find adequate learning material, compelling learning tasks, templates, and guide for the development of cognitive skills [21]. The focus is shifting from the educator directed instruction to a learner centered approach: the learner is at the center of the learning process. This yields that the learning material and tasks should be 
adequate for each learner profile, and that the learner should be guided through the material and tasks so as to achieve the learning goal.

The goal of this research is to enhance the reading comprehension of poor comprehenders. In order to do so, TERENCE system has being developed following the evidence-based design (EBD) and UCD [16], by involving a relevant number of real learners in the project and educators as is depicted in Fig. 1.

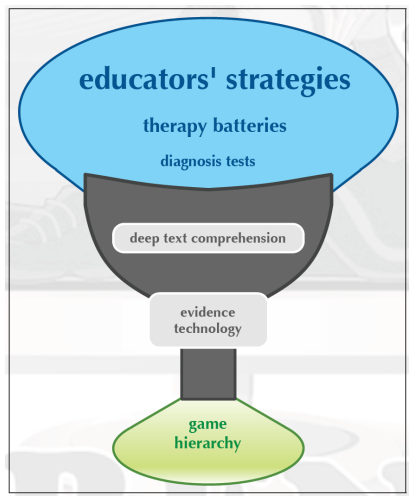

Fig. 1 The UCD and EBD design process of the TERENCE smart games

Data have been collected and analysed through user centred design methods, and then filtered through evidence-based sieves. The strategies of the educators can be framed in the three stages of the hermeneutic cycle explained in [20]. In particular, the explanatory stage can be broken down into the following reading interventions, done in class, mainly using question-answering and drawing:

1. the entire text is discussed with the learners, analysing the vocabulary unknown to the learners and paraphrasing the text;

2. the story is broken down into a sequence of episodes, if possible referring to the story grammar, that is, the story setting, the initiating episode, the culminating episode, the resolving episode, and the final episode;

3. finally, the time, the space and the characters of the story episodes are analysed together.

All the aforementioned interventions were considered for writing the requirements for the TERENCE game design. Constraints of the project triggered a prioritisation of the requirements which led in that visual aids were selected mainly for their expected efficacy for the pedagogy plan, according to the available empirical evidence: they should guide the child to better recall and correlate the information acquired reading the story via adequate visual representations. The effective interventions relevant for the TERENCE design have thus been hierarchically organised in levels according to their main pedagogical goal:

1. time: interventions for reasoning about temporal relations between events of the story, purely sequential (before-after) or not (all the others); 
2. causality: interventions concerning causal-temporal relations between events of the story, namely, the cause of a given event (cause), the effect (effect), or the cause- effect relations between two events (cause-effect);

3. characters: interventions concerning characters, namely, who is the agent of a story event (who), what does a character in the story (what).

The context of use was thoughtfully analysed and specified for characterising the users of TERENCE, and hence stirring the design of the entire system. In this manner, the learning material and tasks were designed so as to be adequate to the real TERENCE learners. TERENCE learning material is made of stories and games for primary school children. Stories are organised in books, and games are distinguished into relaxing games, for relaxing the learners, and smart games, for assessing and stimulating reading comprehension. Each story is related to a set of c.a 2 relaxing games, and a set of c.a 15 smart games.

The EBD practice of the experts responsible for the pedagogical plan requires three main learning tasks in relation to the learning material of the system: (i) reading stories; (ii) playing with smart games for stimulating inference-making about stories; and (iii) playing with relaxing games for relaxing and motivating the learners. The pedagogical goal of relaxing games is to stimulate visuo-perceptual skills [14], instead smart games are designed to stimulate the recall and the correlation of the information acquired while reading a story.

Both smart and relaxing games are effective to provide a playful environment. When learning takes place in a playful environment, learning involves the learner actively and it increases his or her motivation and engagement. For this reason, an accurate stimulation plan has been carefully designed according to various experts' feedback. The experts, in view of their evidence-based experience with stimulation plans for children, suggested to focus on specific types of stimuli, namely, inference-making about events and their relations, in order to train the learners on this within the stimulation plan. Given that inference-making is the specific focus of the project, interventions were related to inference making about stories, and to deep text comprehension more in general.

The results of the stimulation plan setted specific requirements on the design of the TERENCE smart games, related to Time, Accuracy and Level of difficulty. As a result of these requirements, relaxing games and smart games have been designed as follows:

- Relaxing games are modeled on familiar games and serve to make the stimulation plan more appealing to the learner. Each type of relaxing game aims at stimulating a type of visio-perceptual interaction used in smart games that the TERENCE learners are unlikely to have [14]. Therefore, they relax and motivate the learners to use the TERENCE system within the stimulation plan, after or before playing with smart games.

- Smart games serve to guide the child to better recall and correlate the information acquired reading the story. Moreover, according to the performances of the learners over the smart games, the adaptive module can decide whether to move the learner from one story level to another. Therefore smart games lay at the core of the stimulation plan. 
To summarise, according to the experts of the stimulation plan, TERENCE should not propose games concerning causes to children with very low reading comprehension skills, and should avoid games that are likely to be too unchallenging, like who-questions and what-questions, with children with good reading comprehension skills. In the case of children with very low skills, too demanding games can easily lead to frustration whereas, in the case of children with good skills, unchallenging games can easily lead to boredom.

\section{Characterisation of Learners for Playing Tasks}

By using the UCD, we extensively and deeply analysed the context of use and the learners' requirements, thereby specifying classes of learners for the system. The learning material and tasks of the system were designed for those classes of learners. The first part of this section outlines the type of data collected and analysed for specifying the classes of users. The second part outlines the type of data collected and analysed for designing the learning material and the learning tasks.

\subsection{Classes of Learners}

The specific goal of the project distinguishes the two classes (deaf and hearing learners). These classes were refined on the basis of the results of the analysis of data for the context of use and user requirements. Such data have been gathered via a mix of expert-based method inquiries and user-based method inquiries. The learners involved were about 300 in Italy and about 300 in the UK; the educators involved were about 50 in Italy and about 30 in the UK. Learners are currently represented by five classes in Italy and four classes in UK [14]. The most significant features related to the characteristics of the users and considered for deriving the TERENCE classes are biographical information, personality and usage of technology. All the classes and the features used for deriving the TERENCE classes were then specified using personas, which are explained in [2] [14].

\subsection{Playing Tasks}

All data for the game requirements have been gathered through UCD methods, the results of which are reported in [18] as tasks. In particular, the data for relaxing games are popular causal video games which is a video-game meant for casual gamers who come across the game and can get into the gameplay almost immediately. This means that the causal game has usually simple rules and usually it can be played everywhere, anytime and with any device. The data for smart games are mainly diverse reading strategies by pedagogy experts working as therapists with poor comprehenders, cognitive psychologists or educators. The TERENCE smart games were then layered into similar levels as the previous interventions, that is, smart games at the entry level for reasoning about characters, games at the intermediate level for time, and games at the top level concerning causality. 
The following section delves into how the design and development of the smart and relaxing games is carried out via the TERENCE framework.

\section{The Design and Development of Games via TERENCE Framework}

According to the game design guidelines [1] for specifying the gameplay of the TERENCE games we analysed the data for the gameplay of each TERENCE game, then we abstracted the common characteristics in the TERENCE game framework presented in Table 1.

Table 1 The TERENCE game framework

\begin{tabular}{|c|c|}
\hline Name & name of the game \\
\hline Instructions & $\begin{array}{l}\text { instructions concerning the game, for the learner: specific to the game in- } \\
\text { stance; motivational; concerning the rules }\end{array}$ \\
\hline Choices & the choices available to the learner; their availability is state depen- dent \\
\hline \multirow[t]{2}{*}{ Solutions } & Correct \\
\hline & which choices are correct solutions which choices are wrong solutions \\
\hline \multirow[t]{2}{*}{ Consistency f } & correct \\
\hline & a yes-message for correct solutions a no-message for wrong solutions \\
\hline \multirow[t]{2}{*}{ Explanatory f. } & correct \\
\hline & $\begin{array}{ll}\text { explanatory message for correct so- } & \text { explanatory message for wrong solu- } \\
\text { lutions } & \text { tions }\end{array}$ \\
\hline Solution $\mathbf{f}$. & a message consisting in the correct solution \\
\hline $\begin{array}{l}\text { Smart points (e.g., } \\
\text { coins) }\end{array}$ & $\begin{array}{l}\mathbf{K} . P(\theta) \text {, where } \theta \text { is the underlying ability of the learner for the game, and } \mathbf{K} \\
\text { is a constant ranging over natural numbers }\end{array}$ \\
\hline $\begin{array}{l}\text { Relaxing points } \\
\text { (e.g., starts) }\end{array}$ & $\mathrm{M}$, that is, a natural number from 1 up to $\mathrm{N}$ \\
\hline Avatar & the states of the avatar \\
\hline Time & resolution time $\mathbf{t r}$ \\
\hline Rules & $\begin{array}{l}\text { the rules for the game mechanics, specifying the states of the system, the learners' ac- } \\
\text { tions and the transitions from state to state through the learner's actions }\end{array}$ \\
\hline
\end{tabular}

The framework serves to specify in a structured manner the above data for the gameplay of the TERENCE smart and relaxing games, essentially, through a timed transition system, with states of the system, and transitions labelled by the player's actions and time constraints.

In the following, we firstly present the common elements of framework for relaxing of smart games:

- The instructions for the game are questions specific to the game instance; of motivational type and usually related to the learner avatar; concerning the rules. 
- The available choices may change from state to state of the game: at the beginning all the choices are available; when the play starts, some choices may become unavailable. The solutions for the game list the choices or their combination that form a correct solution to the game (correct), and those that do not (wrong).

- The feedback for the game is specialised into a consistency feedback (yes, no), an explanatory feedback for finding a correct solution (for correct) or for spotting what is wrong in the current solution (for wrong), and a solution feedback (the correct solution).

- The states of the avatar in the gameplay are of two kinds: happiness for the correct solution, disappointment for the wrong solution. The resolution time is a constant.

The Smart points are the points a learner with a specific reading comprehension level can gain in a smart game. These points can be calculated using the IRT [7], so that the more difficult a game is (assessed to be) for a learner, the more points the learner can gain in resolving correctly that game. Relaxing games have relaxing points instead of smart points. Relaxing points should be easy to cumulate, so as to motivate the learner to keep on playing and, in so doing, earning attributes for the avatar.

Now, like points, rules are different for smart games and relaxing games.

- Smart rules, the pedagogical plan establishes requirements for the actions that the learner can take, the states the system can be in, and constraints on them. In the following, we sketch the actions, the states and the constraints for smart games:

The plan also recommends diverse types of feedback if the learner makes a wrong choice: first, a no-consistency feedback for signaling that the solution is wrong, and then an explanatory feedback are given.

The plan also suggests a solution feedback if the leaner chooses no solution within the resolution time or the number of wrong solutions overcomes the wrong attempts limit.

The main states the system can be in are as follows:

(i) the initial state, in which the learner score $s$ and resolution time $t$ are set to 0 , the smart points for the learner are computed as a function of the learner ability in the game, all the choices are set as available, and the number of wrong answers is set to 0 ;

(ii) a terminal state reachable via a correct action, in which a yes-consistency feedback is given, the score is displayed and the avatar is in the happy status;

(iii) a terminal state reachable via a skip action, in which the solution feedback is given, the null score is displayed and the avatar is in the displeased status;

(iv) a state, reachable via a wrong action, in which a no-consistency feedback is given, an explanatory feedback is given, the set of available choices is updated, and the number of wrong answers is updated; and

(v) a terminal state reachable via a wrong action, in which the no-consistency feedback is given, the solution feedback is given, the null score is displayed and the avatar is in the displeased status. 
- Relaxing rules have the same rules as well, based on common rules for casual games.

In the initial state, the score and resolution time are set to 0. From any nonterminal state, we can have the following: let $\mathrm{N}$ be the number of relaxing points that can be cumulated in a relaxing game, if the score is less than $\mathrm{N}$ and, within the game's resolution time, the learner chooses a correct solution, then the system shows the yes-consistency feedback, and the score gets increased by 1. But, if the learner chooses a wrong solution, then the system shows the noconsistency feedback, the game terminates and the system shows the disappointed avatar; otherwise, the system terminates the game, shows the score and the happy avatar.

\subsection{Conclusion and Game Prototype}

In this paper we explained how the playing material and tasks of TERENCE are designed and developed on top of an extensive analysis of the requirements of the TERENCE learners based on (EBD) and UCD. This study finalizes with the development of the prototypes of smart games, like the one in Fig. 2. The development procedure, from the framework via the visual template to the prototypes is reported in [3].

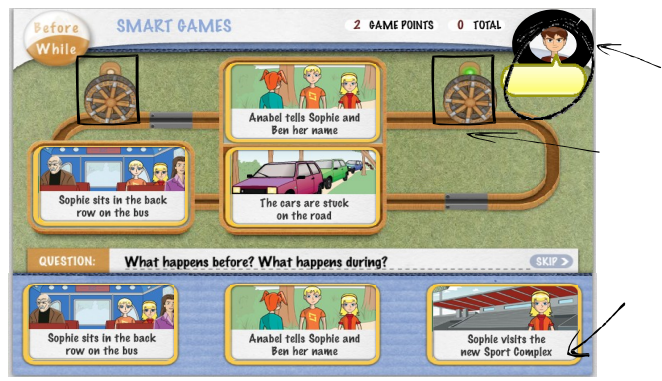

Fig. 2 An instance of a prototype of a before-while smart game

Acknowledgements. The authors' work was supported by TERENCE project, funded by the EC through the FP7 for RTD, Strategic Objective ICT-2009.4.2, ICT, TEL. The contents of the paper reflects only the authors' view and the EC is not liable for it. Gennari work was also funded through the CRESCO and DARE projects, financed by LUB and the Province of Bozen-Bolzano.

\section{References}

1. Adams, E.: Fundamentals of Game Design. New Riders (2010)

2. Alrifai, M., de la Prieta, F., Di Mascio, T., Gennari, R., Melonio, A., Vittorini, P.: The Learners' User Classes in the TERENCE Adaptive Learning System. In: Proc. of the ICALT 2012 (2012) 
3. Alrifai, M., Gennari, R.: Deliverable 2.3: Game Design. Tech. Rep. D2.3, TERENCE project (2012)

4. Alrifai, M., Gennari, R., Tifrea, O., Vittorini, P.: The user and domain models of the TERENCE adaptive learning system. In: Vittorini, P., Gennari, R., Marenzi, I., de la Prieta, F., Rodríguez, J.M.C. (eds.) International Workshop on Evidence-Based TEL. AISC, vol. 152, pp. 83-90. Springer, Heidelberg (2012)

5. Alrifai, M., Gennari, R., Vittorini, P.: Adapting with evidence: The adaptive model and the stimulation plan of TERENCE. In: Vittorini, P., Gennari, R., Marenzi, I., de la Prieta, F., Rodríguez, J.M.C. (eds.) International Workshop on Evidence-Based TEL. AISC, vol. 152, pp. 75-82. Springer, Heidelberg (2012)

6. Arfe, B.: Deliverable 2.2: Repository of Stories. Tech. Rep. D2.2, TERENCE project (2012)

7. Baker, F., Seock-Ho, K.: Item Response Theory. Dekker Media (2012)

8. Brusilovsky, P.: Adaptive hypermedia: From intelligent tutoring systems to web-based education. In: Gauthier, G., VanLehn, K., Frasson, C. (eds.) ITS 2000. LNCS, vol. 1839, pp. 1-7. Springer, Heidelberg (2000)

9. Brusilovsky, P., Karagiannidis, C., Sampson, D.: Layered evaluation of adaptive learning systems. International Journal of Continuing Engineering Education and Lifelong Learning 1(14), 402-421 (2004)

10. De la Prieta, F., Di Mascio, T., Gennari, R., Marenzi, I., Vittorini, P.: The TERENCE Smart Games: Automatic Generation and Supporting Architecture. In: Proc. of the PDSG 2012 Workshop co-located with EC-TEL 2012. CEUR-WS (2012)

11. Duffy, T.M., Cunningham, D.J.: Constructivism: Implications for the design and delivery of instruction. In: Jonassen, D.H. (ed.). Simon \& Schuster Macmillan, New York (1996)

12. Mascio, T.D.: First User Classification, User Identification, User Needs, and Usability goals, Deliverable D1.2. Tech. rep., TERENCE project (2012)

13. Nanjappa, A., Grant, M.M.: Constructing on constructivism: The role of technology. Electronic Journal for the Integration of Technology in Education 2 (2003)

14. Norman, D.: The design of everyday things. Doubleday, New York (1998)

15. Rizzo, R.: Multimodal and Multimedia aspects of English Language Teaching and Studies in Italian Universities. Ibis, Como (2009)

16. Slegers, K., Gennari, R.: Deliverable 1.1: State of the Art of Methods for the User Analysis and Description of Context of Use. Tech.Rep.D1.1, TERENCE project (2011)

17. Valeriani, A.: Ermeneutica retorica ed estetica nell'insegnamento verso l'oriente del testo. Andromeda (1986)

18. Yelland, N., Masters, J.: Rethinking scaffolding in the information age. Computers and Education 48, 362-382 (2007) 